\title{
Predictors of Acceptance and Rejection of Online Peer Support Groups as a Digital Wellbeing Tool
}

\author{
John McAlaney ${ }^{1}$, Manal Aldhayan ${ }^{1}$, Mohamed Basel Almourad ${ }^{2}$, Sainabou Cham ${ }^{1}$, \\ Raian $\mathrm{Ali}^{3}$ \\ ${ }^{1}$ Faculty of Science and Technology, Bournemouth University, UK \\ \{jmcalaney, maldhayan, scham\}@bournemouth.ac.uk \\ ${ }^{2}$ College of Technological Innovation, Zayed University, UAE \\ basel.almouradezu.ac.ae \\ ${ }^{3}$ Information and Computing Technology Division, College of Science and Engineering, \\ Hamad Bin Khalifa University, Qatar \\ raali2@hbku.edu.qa
}

\begin{abstract}
Digital media usage can be problematic; exhibiting symptoms of behavioural addiction such as mood modification, tolerance, conflict, salience, withdrawal symptoms and relapse. Google Digital Wellbeing and Apple Screen Time are examples of an emerging family of tools to help people have a healthier and more conscious relationship with technology. Peer support groups is a known technique for behaviour change and relapse prevention. It can be facilitated online, especially with advanced social networking techniques. Elements of peer support groups are being already embedded in digital wellbeing tools, e.g. peer comparisons, peer commitments, collective usage limit-setting and family time. However, there is a lack of research about the factors influencing people acceptance and rejection of online peer support groups to enhance digital wellbeing. Previous work has qualitatively explored the acceptance and rejection factors to join and participate in such groups. In this paper, we quantitatively study the relationship between culture, personality, self-control, gender, willingness to join the groups and perception of their usefulness, on such acceptance and rejection factors. The qualitative phase included two focus groups and 16 interviews while the quantitative phase consisted of a survey (215 participants). We found a greater number of significant models to predict rejection factors than acceptance factors, although in all cases the amount of variance explained by the models was relatively small. This demonstrates the need to design and, also, introduce such technique in a contextualised and personalised style to avoid rejection and reactance.
\end{abstract}

Keywords: Online Peer Groups, Digital Addiction, Digital Wellbeing, Behavioural Change

\section{Introduction}

Digital media including social networks, gaming and online shopping have various benefits and represent an integral part of modern society. Such media empower social connectedness, information exchange and freedom of information exchange introduc- 
ing a new lifestyle and concepts such as digital humanity and digital citizenships. However, some compulsive and obsessive usage styles and over-reliance on digital media can lead to negative consequences such as reduced involvement in real-life communities and a lack of sleep [1]. Some usage styles can be seen as addictive meeting common criteria of behavioural addiction such as salience, conflict, mood modification, and relapse $[2,3]$.

There is a limited number of preventative, control and recovery mechanisms available for Digital Addiction (DA). Although the problematic relationship with technology has been recognised in a wide range of literature, DA is still not classified as a mental disorder in the latest 5th edition of the Diagnostic and Statistical Manual of Mental Disorders (DSM 5). Recently, in 2018, the World Health Organization recognised Gaming Disorder, which represents a significant step is searching for preventative and recovery mechanisms. Most of the existing research on DA focuses on the reasons for people to become overly reliant on social media and the relationship of that with factors such as personality traits [4]. Few works have placed software design at the centre of the DA problems, both in facilitating and also in combatting DA, e.g. the digital addiction labels and the requirements engineering for digital well-being requirements in $[6,7]$.

With the advances in sensing and communication technology and internet connectivity, there has been a proliferation of software and smartphone applications to assist with behavioural change. It is still questionable whether these solutions are effective and whether we understand the acceptance and rejection factors from the users' perspective. The perception of their role and trustworthiness of such proposed solutions has changed following some failures and the recognition of associated risks [8].

Linking the intention to change behaviour with the act of doing so is the main purpose of behaviour change theories [5]. Peer support groups are one of the approaches to behaviour change which can be utilised to combat addictive behaviours by providing support and helping in relapse prevention [9]. Peer support groups consist of people sharing similar interests and in view of supporting and influencing each other's behaviour towards achieving common goals [10]. Alrobai et al. [13] focused on the processes involved when running the group, e.g. the roles involved in doing so and the steps to be taken to prevent relapse. Aldhayan et al. [18], explored the acceptance and rejection factors of online peer support groups by people with DA. This exploration was meant to inform the strategies used to introduce such online peer group software, as well as the configuration and governance processes of their online platform.

Hsiao Shu and Huang [17] explored the relationships between personality traits and compulsive usage of social media apps, and showed that extraversion, agreeableness, and neuroticism have significant effects on such compulsive usage. Being an online social technique for behaviour change itself, acceptance and rejection of peer support groups could be in turn subject to such personal and environmental factors. In this paper, we study the effect of personality traits, self-control, gender, and perception of usefulness, willingness to join and culture (comparing UK to Middle Eastern users) on the acceptance and rejection factors of online peer support groups. To achieve this target, we designed a survey around the acceptance and rejection factors reported in [18] and derived from two focus groups and 16 interviews. The survey 
also consisted of various demographics questions and measures for personality [20] and self-control [19]. We collected 215 completed responses. We report on the statistical analysis results and discuss their implications on the design of future online peer support groups to combat DA.

\section{Research Method}

We adopted a mixed-methods approach which consisted of an initial qualitative phase followed by a quantitative one. The participants in both phases self-declared as experiencing problematic digital behaviour and wellbeing issues.

\subsection{Qualitative phase: exploring acceptance, rejection and governance}

We conducted a focus group study of two sessions. The first session aimed at getting insights around how online peer groups are perceived by people self-declaring to have DA and what they wished to see in it. The second focus group served the purpose of identifying the design features of an online peer group platform. For this reason, mock interfaces were made available to the second session participants based on the results of the first focus group. The participants were asked about opinions regarding the mock design and to amend them if needed. The two focus group sessions were conducted with the same six university students; three male and three females, aged between 20 and 26 . The participants were a social group in real life, and this was beneficial as it removed concerns regarding trust and privacy during the discussion process. We performed a thematic analysis [12] on the data collected through the sessions. This analysis revealed main factors concerning the acceptance and rejection of this approach as well as its governance styles and process.

The objective of the interview stage was to explore in-depth the acceptance and rejection factors and the variability space of designing online peer groups platforms so that we can accommodate different users' preferences and governance styles. The interview questions were based on the acceptance and rejection factors explored in the focus groups as well as five themes related to governance, including group moderation, feedback and monitoring, membership and exit protocol. We conducted 16 interviews with students who self-declared to have a wellbeing issue around their digital behaviour, e.g., obsessive or compulsive use. The sample consisted of 8 males and 8 females, aged between 18 and 35. Each interview lasted between 30 and 40 minutes. The interviews were transcribed and analysed via thematic analysis [12].

\subsection{Quantitative phase: confirmation, personal and environmental influences}

This phase was based on a survey that reflected the interview themes, i.e. the acceptance and rejection themes as well as governance themes such as moderator role, feedback, membership and exit procedure. The survey was disseminated both online and in person. A $£ 5$ incentive was offered to respondents given the lengthy nature of the survey. We collected 215 completed responses; 105 participants (49\%) identified as male and 109 participants (50\%) identified as female, with the remaining $1 \%$ preferring not to answer on the gender question. The participants were 17 to 55 years old. 
The survey started with a validation question of whether a participant has wellbeing issues as a precondition to take part.

To study the effect of personal and environmental factors on the acceptance and rejection factors, the survey included questions around six factors which were gender (male/ female); country/culture (UK/ Middle East); perceived usefulness of peer support groups; willingness to join a peer support group; five personality traits [20] (extraversion, agreeableness, conscientiousness, neuroticism and openness); and selfcontrol [19]. We disseminated the survey mainly in the UK, the Kingdom of Saudi Arabia and Syria. We collected 104 completed surveys from KSA and Syria, and 85 from the UK. This allowed us to study statistically whether there was a difference between Middle Eastern culture (KSA and Syria) and Western Culture (UK).

\section{$3 \quad$ Acceptance and Rejection Factors}

The factors which affect users' acceptance and rejection of online peer support groups to combat DA are presented in Tables 1 and 2, respectively. The elaborated descriptions of themes A1 to A4 and R1 to R4 can be found in [18]. Further analysis of the data revealed another theme, which is A5.

Table 1 Online Peer Support Groups to Combat Digital Addiction: Acceptance Factors

\begin{tabular}{|l|l|}
\hline Acceptance Theme & Sub-themes \\
\hline $\begin{array}{l}\text { [A1] Accepting online } \\
\text { peer groups as an enter- } \\
\text { tainment auxiliary }\end{array}$ & $\begin{array}{l}\text { [A1.1] Provide awards: gamification of performance } \\
\text { [A1.2] Peer comparison: to see how I and others do } \\
\text { [A1.3] Goal achievement: rewards, information and graphs of } \\
\text { my progress towards the goal }\end{array}$ \\
\hline $\begin{array}{l}\text { [A2] Accepting online } \\
\text { peer groups as a DA } \\
\text { awareness tool }\end{array}$ & $\begin{array}{l}\text { [A2.1] Self-Monitoring: show actual usage and performance } \\
\text { [A2.2] Peer comparison: benchmarking through others } \\
\text { [A2.3] Goal achievement: awareness of how I am achieving } \\
\text { goals }\end{array}$ \\
\hline $\begin{array}{l}\text { [A3] Accepting online } \\
\text { peer support groups as an } \\
\text { educational tool }\end{array}$ & $\begin{array}{l}\text { [A3.1] Peer learning: learning from others how to improve } \\
\text { acting as moderator } \\
\text { [A3.3] Set up goals: learning how to set up SMART goals }\end{array}$ \\
\hline $\begin{array}{l}\text { [A4] Accepting online } \\
\text { peer support groups as a } \\
\text { prevention tool }\end{array}$ & $\begin{array}{l}\text { [A4.1] Peer feedback: alert/feedback through peer feedback } \\
\text { [A4.2] Moderator feedback: alert/feedback by a moderator } \\
\text { [A4.3] Authority: steps and restrictions set by a moderator }\end{array}$ \\
\hline $\begin{array}{l}\text { [A5] Accepting online } \\
\text { peer support groups as a } \\
\text { support tool }\end{array}$ & $\begin{array}{l}\text { [A5.1] Provide advice: by experienced moderator; alternatives } \\
\text { lifestyle } \\
\text { [A5.2] Emotional support: when struggling to avoid relapse } \\
\text { [A5.3] Feedback: when performing well and under-performing, } \\
\text { sending warnings }\end{array}$ \\
\hline
\end{tabular}

Table 2 Online Peer Support Groups to Combat Digital Addiction: Rejection Factors

\begin{tabular}{|l|l|}
\hline Rejection Theme & Sub-themes \\
\hline $\begin{array}{l}\text { [R1] Rejecting online } \\
\text { peer support groups when } \\
\text { seen as intimidation tool }\end{array}$ & [R1.1] Negative feedback: dismissive feedback when failing \\
\hline [R2] Rejecting online & [R2.1] Being overly judged by a moderator \\
\hline
\end{tabular}




\begin{tabular}{|l|l|}
\hline $\begin{array}{l}\text { peer support groups when } \\
\text { seen as overly judgmental }\end{array}$ & {$[\mathrm{R} 2.2]$ Being judged by peers, known and unknown in person } \\
\hline $\begin{array}{l}\text { [R3] Rejecting online } \\
\text { peer supports group when } \\
\text { hosting unmanaged in- } \\
\text { teractions }\end{array}$ & {$[\mathrm{R} 3.1]$ Weak management } \\
[R3.2] Large group size \\
\hline $\begin{array}{l}\text { [R4] Rejecting online } \\
\text { peer groups due to un- } \\
\text { clear membership proto- } \\
\text { col }\end{array}$ & $\begin{array}{l}\text { [R4.1] Relatedness: group including relatives and friends } \\
\text { tions on exiting the group without considering others }\end{array}$ \\
\hline
\end{tabular}

\section{Personal and Cultural Effects on Acceptance and Rejection}

The survey questions around acceptance and rejection can be found in Appendix A. A Likert scale indicating level of agreements was used for each of the statements under each theme. A series of linear multiple regressions using the enter method were conducted. In each model the predictors were gender (male/ female); region (UK/ Middle East); perceived usefulness of peer support groups; willingness to join a peer support group; the five personality trait scores of extraversion, agreeableness, conscientiousness, neuroticism and openness; and self-control score. For each model, the outcome measure was the individual questions used to measure attitudes relating to the acceptance and rejection factors of online peer groups, as identified within the description of each model result in the section below. Multicollinearity diagnostics were conducted prior to the analysis to determine the suitability of conducting multiple regressions.

\subsection{Effects on acceptance factors}

[A1] Accepting online peer groups as an entertainment auxiliary. Three models under this category were non-significant, which were [A1.1a] Awards when achieving behavioural targets, e.g. points, badges, etc.; [A1.1b] Awards when making progress towards the behavioural target; [A1.3] Information and graphs how I am progressing to keep me engaged. The model for [A1.2] Peer comparisons, i.e. to see how I and others are performing was significant, predicting $12 \%$ of the variance $\left(\mathrm{R}^{2}=.12\right.$, $\mathrm{F}(10,159)=2.16, \mathrm{p}<.05)$. Of this extraversion was the only significant predictor $(\beta=$ .12 ), with an increase in extraversion being associated with an increase in agreement towards this statement.

[A2] Accepting online peer support groups as an awareness tool. The first model under this category was significant, which was [A2.1] Self-Monitoring, e.g. showing your hourly, daily and weekly performance and progress indicator $\left(\mathrm{R}^{2}=.11\right.$, $\mathrm{F}(10,159)=1.90, \mathrm{p}<.05)$, accounting for $11 \%$ of the variance. Within this model the two significant predictors were extraversion $(\beta=0.09)$ and neuroticism $(\beta=.07)$. In each as the level of the personality trait increased there was an increase in agreement towards this statement. The other two models under this category were not significant. 
These were [A2.2] Peer comparisons, e.g. comparing you to other members in the group who have similar profile and level of problem; [A2.3] Awareness on goal setting, e.g. how to set and achieve goals, and how to avoid deviation from the plan you sat to achieve them.

[A3] Accepting online peer support groups as an educational platform. None of the models under this category was significant. These were [A3.1] Environment to learn from a peers, e.g., by sharing real-life stories and successful strategies around the wellbeing issue; [A3.2a] Environment to learn from experienced moderators, e.g. best practice around the wellbeing issue; [A3.2b] Environment where I can learn through acting as a mentor, i.e. when advising other members and when having to moderate the group; [A3.3] Environment to learn how to set up achievable and effective goals and their plans. This suggests that as an education tool, peer support groups acceptance is not affected by differences in personal and environmental factors.

[A4] Accepting online peer support groups as a digital addiction prevention tool. None of the models under this category was significant, which were [A4.1] Feedback messages sent by peers about performance and wellbeing goals. [A4.2] Guidance, feedback and information sent by moderators based on performance and achieving wellbeing goals; [A4.3] Steps, restrictions and plans set by an authorised moderators, e.g. game usage limit for compulsive gamers;

[A5] Accepting online peer support groups as a support tool. The first model for [A5.1a] Environment to provide experienced moderators who are able to provide advice and guide members to manage the wellbeing issue was significant $\left(\mathrm{R}^{2}=.12\right.$, $\mathrm{F}(10,159)=2 . .01, \mathrm{p}<.05)$, accounting for $12 \%$ of the variance. The only significant predictor was neuroticism $(\beta=.07)$, with an increase in this personality trait being associated with an increase in acceptance towards this statement. The rest of regression models under this category were not significant. These were [A5.1b] Environment to suggest alternative activities to replace and distance myself from the negative behaviours and enhance wellbeing; [A5.2] Environment to provide emotional support, e.g. when struggling to follow the healthy behaviour; [A5.3a ] Environment to get positive and motivational feedback when performing well; [A5.3b] Environment to get positive and motivational feedback even when failing to achieve targets; [A5.3c] Environment to issue warning feedback when members performance and interaction are not right;. This again suggests that influences are limited when peer groups are seen as knowledge and advice source.

\subsection{Effects on rejection factors}

[R1] Rejecting online peer support groups when seen as an intimidation tool. The model for [R1.1a] I reject a group with negative feedback, e.g. you have repetitively failed in achieving your target, this is the 5th time this month was significant $\left(\mathrm{R}^{2}=\right.$ $.11, \mathrm{~F}(10,159)=2, \mathrm{p}<.05)$, accounting for $11 \%$ of the variance. Within the model, the only significant predictor was openness $(\beta=-.16)$. As such as there was an increase 
in agreeableness there was a decrease in acceptance of this statement. The model for [R1.2b] I reject a group with harsh feedback, e.g. Your interaction with peers shows anti-social and disruptive patterns. You have been reported for annoying others was significant $\left(\mathrm{R}^{2}=.12, \mathrm{~F}(10,159)=2.3, \mathrm{p}<.05\right)$, accounting for $12 \%$ of the variance. Within the model the only significant predictor was gender $(\beta=.4)$. This meant that female participants were more likely to accept this statement. The model for [R1.2] I reject a group with harsh penalties e.g. banning from the group for a period of time if I repetitively forget my target was significant $\left(\mathrm{R}^{2}=.16, \mathrm{~F}(10,159)=3.1, \mathrm{p}<.05\right)$, accounting for $16 \%$ of the variance. Within the model the significant predictors were agreeableness $(\beta=.13)$, neuroticism $(\beta=.15)$ and self-control $(\beta=-.04)$. As such as agreeableness and neuroticism increased there was an increase in acceptance of this statement, but as self-control increased there was a decrease in acceptance of this statement.

[R2] Rejecting online peer support groups when seen as overly judgmental. Three of the regression models under this category were not significant, which were [R2.1] I reject a group if the group moderator judges my performance and interaction frequently, even if this is for my benefit [R2.2a] I reject a group if I am judged by peers who are only online contact, e.g. not real-life contacts; [R2.2c] I reject a group if the judgement online expands to other life aspects by peers who are real-world contacts;. The model for [R2.2b] I reject a group if I am judged by online peers who are also real-world contacts was significant $\left(\mathrm{R}^{2}=.14, \mathrm{~F}(10,159)=2.6, \mathrm{p}<.01\right)$, accounting for $14 \%$ of the variance. Within the model, the only significant predictor was gender $(\beta=$ .56). This meant that female participants were more likely to accept this statement.

[R3] Rejecting online peer supports group when hosting unmanaged interactions. The model for [R3.1a] I reject a group with a weak moderator, e.g. unable to stop or ban members who are not adhering to the group norms was significant $\left(\mathrm{R}^{2}=.12\right.$, $\mathrm{F}(10,159)=2.1, \mathrm{p}<.05)$, accounting for $12 \%$ of the variance. Within the model, conscientiousness was the only significant predictor $(\beta=0.14)$, with an increase in this trait being associated with an increase in agreement with this statement. The model for [R3.1b] I reject a group which allows a loose and relaxed rules e.g. accepting conversations and interactions that are not related to the wellbeing issue, was significant $\left(\mathrm{R}^{2}=.13, \mathrm{~F}(10,159)=2.4, \mathrm{p}<.05\right)$, accounting for $13 \%$ of the variance. Within the model the predictors of conscientiousness $(\beta=0.14)$ and openness $(\beta=-.2)$ were both significant, with an increase in conscientiousness being associated with an increase in acceptance of this statement. In contrast an increase in openness was associated with a decrease in this acceptance of this statement. The remaining model of [R3.2] I reject a group with a large size as it may not feel as a coherent group was not significant.

[R4] Rejecting online peer groups due to unclear membership protocol. None of the models under this category was significant, which were [R4.1a] I reject a group which allows friends in real-life to join; [R4.1b] I reject a group which allows family members to join; [R4.2a] I reject a group when members can leave the group anytime 
without giving notice and explanation; [R4.2b] I reject a group when there are conditions to exit the group, e.g. to tell the moderator in advance.

\subsection{Discussion}

In terms of acceptance factors, the majority of regression models were not significant, with those that were only explaining a relatively small amount of the variance. The significant predictors with such models were primarily personality traits, such as extraversion and neuroticism. These occurred in the direction that could be as expected, such as for example, an increase in extraversion being associated with acceptance of a peer group to increase engagement in managing a wellbeing issue.

There were a greater number of significant regression models under the rejection factors, although again when these were significant, they only accounted for a relatively low amount of the variance. The greater number of significant models and predictors in relation to rejection factors compared to acceptance may be a reflection of the reactance effect [15], in which individuals respond negatively to being told that they are not permitted to do something. Similar to the significant acceptance model personality traits tended to be amongst the significant predictors. Gender was a significant predictor in several models relating to group judgement, with female participants being found to be more likely to reject statements that involved the possibility of social judgement. Research into the gender and the use of peer groups has found that the relationship between these can be complex [16]; however this result could be argued to be consistent with the broad finding that females make greater use of social support structures. This is because a peer group situation that includes explicit and trackable judgement of others may be perceived to be a threat to group harmony, and therefore something which may be likely to undermine or damage that social support network.

Culture was not a significant predictor in any of the significant regression models. This was unexpected, as there are several cultural dimensions that could be relevant to peer group structure and function. This includes dimensions such as power distance, individualism and uncertainty avoidance [21]. This may suggest that online peer support environments are not subjected to cultural influences in the same way as offline groups, although research in both of these domains is limited. If culture is not an influential factor on acceptance and rejection factors of online peer support groups then this is an important consideration, as it suggests that strategies based around online peer support may be transferable between cultures.

\section{Conclusion and Future Work}

There is increasing societal concerns about the compulsive and excessive use of digital technologies. These same technologies allow for prevention and intervention strategies to be delivered in way that is faster and substantially less costly than traditional strategies, but in order to make this meaningful we must better understand what factors determine the acceptance and rejection of such approaches. In this paper, we studied the effect of several personal and contextual factors on the acceptance and rejection of the online peer support groups as a mechanism to enhance wellbeing. We 
took digital wellbeing as a case study where both the behaviour and the behaviour change share the same medium and where part of the behavior and performance towards behavioural goals and limits can be tracked and monitored in part, i.e. the digital usage. There were fewer differences than what we have expected if we consider similar research in the context of social media. This would mean that online peer support groups, as a special kind of social networks, would need to be thought as a purpose-driven gathering. For example, accepting such a technique as an awareness tool and as an education tool was little affected by personal and cultural differences. We, however indeed noted that rejecting the groups for various reasons including being a medium of unmanaged and loose interaction, with additional risks such as peer interactions tools being used for the purpose of intimidation. This would again mean that people expect such groups to be purpose-driven and reject their instantiation as ordinary social networks. It is important that further research is conducted within this emergent area, to ensure that prevention and intervention strategies are informed by an evidence base.

\section{References}

1. Hampton K, Goulet LS, Rainie L, Purcell K.: Social networking sites and our lives. Pew Internet \& American Life Project 16, 1-85 (2011).

2. Griffiths, M.: A 'components' model of addiction within a biopsychosocial framework. Journal of Substance use10 (4), 191-197 (2005).

3. Widyanto, L. and Griffiths, M.: Internet addiction: a critical review. International Journal of Mental Health and Addiction, 4(1), 31-51(2006).

4. Winkler, A., Dörsing, B., Rief, W., Shen, Y. and Glombiewski, J.A.: Treatment of internet addiction: a meta-analysis. Clinical psychology review 33(2), 317-329 (2013).

5. Webb, T.L., Sniehotta, F.F., Michie, S.: Using theories of behaviour change to inform interventions for addictive behaviours. Addiction. 105, 1879-1892 (2010).

6. Ali, R., Jiang, N., Phalp, K., Muir, S., McAlaney, J.: The Emerging Requirement for Digital Addiction Labels. REFSQ. 9013, 198-213 (2015).

7. Alrobai, A., Phalp, K., Ali, R.: Digital Addiction: A Requirements Engineering Perspective. Requirements Engineering: Foundation for Software Quality. 8396, 112-118 (2014)

8. Alrobai, A., McAlaney, J., Phalp, K. and Ali, R. Exploring the risk factors of interactive ehealth interventions for digital addiction. International Journal of Sociotechnology and Knowledge Development 8 (2), 1-15(2016).

9. Davidson, L., Chinman, M., Kloos, B., Weingarten, R., Stayner, D., Tebes, J.K.: Peer support among individuals with severe mental illness: a review of the evidence. Clinical Psychology: Science and Practice. 6, 165-187 (2006)

10. Alrobai, A., McAlaney, J., Phalp, K. and Ali, R.: Online peer groups as a persuasive tool to combat digital addiction. In International Conference on Persuasive Technology, 288300(2016).

11. Alrobai, A., Dogan, H., Phalp, K. and Ali, R.: Building Online Platforms for Peer Support Groups as a Persuasive Behavior Change Technique. In International Conference on Persuasive Technology, 70-83 (2018).

12. Braun, V., Clarke, V. and Terry, G.: Thematic analysis. Qual Res Clin Health Psychol, 24, 95-114 (2014). 
13. Alrobai, A.: Engineering social networking to combat digital addiction: the case of online peer groups. Doctoral dissertation, Bournemouth University, (2018).

14. Sousa, M.J., Rocha, Á., Game based learning contexts for soft skills development. In World Conference on Information Systems and Technologies, 931-940 (2017).

15. Brehm, S. and J. Brehm, Psychological reactance: A theory of freedom and control. Academic Press New York, NY (1981)

16. Matud, M.P.: Structural gender differences in perceived social support. Personality and Individual Differences. Vol. 35, PP 1919-1929 (2003).

17. Hsiao, K.L., Shu, Y. and Huang, T.C.: Exploring the effect of compulsive social app usage on technostress and academic performance: Perspectives from personality traits. Telematics and Informatics, Vol. 34, PP 679-690 (2017).

18. Aldhayan, M., Cham, S., Kostoulas, T., Almourad, M.B. and Ali, R.: Online Peer Support Groups to Combat Digital Addiction: User Acceptance and Rejection Factors. In World Conference on Information Systems and Technologies, 139-150 (2019).

19. Tangney, June P., Roy F.: Baumeister, and Angie Luzio Boone. "High self-control predicts good adjustment, less pathology, better grades, and interpersonal success. Journal of personality 2 (72), 271-324 (2004).

20. Rammstedt, B. and John, O.P.: Measuring personality in one minute or less: A 10-item short version of the Big Five Inventory in English and German. Journal of research in Personality, 1 (41),203-212 (2007).

21. Hofstede, G.H., G.J. Hofstede, and M. Minkov: Cultures and organizations. Software of the mind. 3rd ed, New York: McGraw-Hill. xiv, 561 (2010).

\section{Appendix A: Survey questions relevant to this paper}

\section{Demographics, perception of peer groups, personality, and self-control questions}

- What is the gender you identify yourself with? Male; Female; Prefer not to say.

- What is your main country?

- How do you see the usefulness of online peer support group as a method to help members in managing their wellbeing issues? Very useful; Useful; Moderately useful; Slightly useful; Not at all useful.

- Would you like to join an online peer support group to help you manage a wellbeing issue? Very likely; Likely; Unlikely; Extremely unlikely.

- 10 Personality questions [20]: How well do the following statements describe your personality? I see myself as someone who: is reserved; is generally trusting; tends to be lazy; is relaxed; handles stress well; has few artistic interests; is outgoing, sociable; tends to find fault with others does a thorough job; gets nervous easily; has an active imagination.

- 13 Self-control questions [19] Using the 1 to 5 scale below, please indicate how much each of the following statements reflects how you typically are: I am good at resisting temptation; I have a hard time breaking bad habits; I am lazy; I say inappropriate things; I do certain things that are bad for me, if they are fun; I refuse things that are bad for me; I wish I had more self-discipline; People would say that I have iron self-discipline; Pleasure and fun sometimes keep me from getting work done; I have trouble concentrating; I am able to work effectively toward long-term goals; 
Sometimes I can't stop myself from doing something, even if I know it is wrong; I often act without thinking through all the alternatives.

\section{Questions about acceptance factors (5 points Likert scale reflecting agreement degree)}

[A1] Online peer support groups method is seen by some as an auxiliary mechanism to ease and add more engagement to the management of the wellbeing issue. Accordingly, the following features will increase my acceptance of them: [A1.1a] Awards when achieving behavioural targets, e.g. points, badges, etc. [A1.1b] Awards when making progress towards the behavioural target. [A1.2] Peer comparisons, i.e. see how I and others are performing. [A1.3] Information and graphs how I am progressing to keep me engaged.

[A2] Online peer groups method is seen by some as an awareness tool to help raise awareness and knowledge about the wellbeing issue and level of the problem. Accordingly, the following features will increase my acceptance of them: [A2.1] Self-Monitoring, e.g. showing your hourly, daily and weekly performance and progress indicator. [A2.2] Peer comparisons, e.g. comparing you to other members in the group who have similar profile and level of problem. [A2.3] Awareness on goal setting, e.g. how to set and achieve goals, and how to avoid deviation from the plan you sat to achieve them.

[A3] Online peer support group method is seen by some as an educational platform to learn how to regulate the wellbeing issue and change behavior. Accordingly, the following features will increase my acceptance of them: [A3.1] Environment to learn from a peers, e.g., by sharing real-life stories and successful strategies around the wellbeing issue. [A3.2a] Environment to learn from experienced moderators, e.g. best practice around the wellbeing issue. [A3.2b] Environment where I can learn through acting as a mentor, i.e. when advising other members and when having to moderate the group. [A3.3] Environment to learn how to set up achievable and effective goals and their plans.

[A4] Online peer support groups method is seen by some as a prevention and precautionary mechanism when the wellbeing issue starts to emerge. Accordingly, the following features will increase my acceptance of them: [A4.1] Feedback messages sent by peers about performance and wellbeing goals. [A4.2] Guidance, feedback and information sent by moderators based on performance and achieving wellbeing goals. [A4.3] Steps, restrictions and plans set by an authorised moderators, e.g. game usage limit for compulsive gamers

[A5] Online peer support groups method is seen by some as a support tool to guide, motivate and encourage the recovery processes of the wellbeing issue. Accordingly, I accept online peer groups as an: [A5.1a] Environment to provide experienced moderators who are able to provide advice and guide members to manage the wellbeing 
issue. [A5.1b] Environment to suggest alternative activities to replace and distance myself from the negative behaviours and enhance wellbeing. [A5.2] Environment to provide emotional support, e.g. when struggling to follow the healthy behaviour. [A5.3a] Environment to get positive and motivational feedback when performing well. [A5.3b] Environment to get positive and motivational feedback even when failing to achieve targets. [A5.3c] Environment to issue warning feedback when members performance and interaction are not right.

\section{Questions about rejection factors (5 points Likert scale reflecting agreement)}

[R1] Online peer groups method is rejected by some as it can be intimidating if used in certain modalities. [R1.1a] I reject a group with negative feedback, e.g. you have repetitively failed in achieving your target, this is the 5th time this month. [R1.1b] I reject a group with harsh feedback, e.g. Your interaction with peers shows anti-social and disruptive patterns. You have been reported for annoying others. [R1.2] I reject a group with harsh penalties e.g. banning from the group for a period of time if I repetitively forget my target.

[R2] Online peer group method is rejected by some when seen as overly judgmental. [R2.1] I reject a group if the group moderator judges my performance and interaction frequently, even if this is for my benefit. [R2.2a] I reject a group if I am judged by peers who are only online contact, e.g. not real-life contacts. [R2.2b] I reject a group if I am judged by online peers who are also real-world contacts. [R2.2c] I reject a group if the judgment online expands to other life aspects by peers who are real-world contacts.

[R3] Peer group is rejected when seen as a medium for a loose and unmanaged interaction. [R3.1a] I reject a group with a weak moderator, e.g. unable to stop or ban members who are not adhering to the group norms. [R3.1b] I reject a group which allows a loose and relaxed rules e.g. accepting conversations and interactions that are not related to the wellbeing issue. [R3.2] I reject a group with a large size as it may not feel as a coherent group.

[R4] Online peer support group method is rejected when the membership protocol is unclear. Please indicate your opinion of the following: [R4.1a] I reject a group which allows friends in real-life to join. [R4.1b] I reject a group which allows family members to join. [R4.2a] I reject a group when members can leave the group anytime without giving notice and explanation. [R4.2a] I reject a group when there are conditions to exit the group, e.g. to tell the moderator in advance. 